ON

THE EFFECTS

or

\title{
LOSS OF BLOOD.
}

By MARSHALI HALI, M.D. F.R.S. Ed. etc.

Read Nov. 23d, 1824.

THE subject of the present essay appears to me to have escaped, in a great measure, the attention both of the physiologist and of the practical physician. The immediate effects of a sudden hæmorrhagy or copious loss of blood are, indeed, sufficiently known; but I am not aware that any author has described with accuracy and distinctness the secondary or more remote effects of loss of blood, in the various circumstances of repetition or continued flow in which it may occur. And yet, when we reflect how often blood-letting is employed as a remedy, and how frequently hæmorrhagy occurs as a disease, it must be considered of great moment, as well as interest, to trace the varied effects of a diminished quantity of blood on the different functions and organs of the animal frame. 
This inquiry possesses a higher interest still, if, as I believe, and hope to explain in the ensuing observations, the more obvious effects of loss of blood are such as suggest the idea of increased power and energy of the system, and lead to an erroneous and dangerous employment of the lancet, when a directly opposite treatment is required. It is now several years since $I$ began the investigation, the results of which I am about to detail. I had for some time contemplated an experimental inquiry, when several opportunities occurred to me, and one especially under extraordinary circumstances, of observing the effects of loss of blood in the human subject; and it will be readily apprehended how much more important it is to have observed the phenomena in question, in instances in which the mental affections and the sensations could be ascertained, as well as the changes in other functions, than merely to have noticed effects obvious to the eye alone.

In stating the results of my observations on the effects of loss of blood, I propose to adopt the following arrangement: I shall treat

I. Of the immediate effects of loss of blood, chiefly syncope, and of the re-action or failure of the vital powers.

II. Of the more remote or cumulative effects of repeated or protracted loss of blood,-or 
Exhaustion; and 1. Of exhaustion with excessivereaction; 2. Of exhaustion with defective re-action; 3. Of exhaustion with sinking.

III. Of the effects of further loss of blood in cases of exhaustion. 1. Of the substitution of syncope for re-action; 2 . Of the transition of the state of re-action into that of sinking; 3. Of sudden dissolution.

IV. Of the influence of various circumstances on the effects of loss of blood. 1. Of age, \&c.; 2. Of disease.

V. Of the effects of loss of blood on the internal organs. 1. The brain; 2. The heart; 3. The lungs; 4. The intestinal canal, \&rc.

I. Of the immediate effects of loss of blood, chiefly syncope, and of the re-action or failure of the vital powers.

The most familiar of the effects of loss of blood is syncope. The influence of posture, and the first sensations and appearances of the patient, in this state, appear to denote that the brain is the organ the function of which is first impaired; the respiration suffers as an immediate consequence; and the action of the heart becomes enfeebled, first from a deficient quantity of blood, and secondly fiom its deficient arterialization. The ca- 
pillary circulation also suffers, and if the state of syncope be long continued, the stomach and bowels become affected. In ordinary syncope from loss of blood, the patient first experiences a degree of vertigo, to which loss of consciousness succeeds. The respiration is affected in proportion to the degree of insensibility, being suspended until the painful sensation produced rouses the patient to draw deep and repeated sighs, and again becoming suspended as before. The beat of the heart and of the pulse is slow and weak; the face and general surface become pale, cool, and bedewed with perspiration; the stomach is apt to be affected with eructation or sickness. On recovery there is perhaps a momentary delirium, yawning, and a return of consciousness; irregular breathing in sighs, and a gradual recovery of the pulse.

In cases of profuse hæmorrhagy the state of the patient varies: there is at one moment a degree of syncope, then a partial recovery. During the syncope the countenance is extremely pallid; there is more or less insensibility ; the respiratory movements of the thorax are at one period imperceptible, and then there are irregular sighs; the pulse is slow, feeble, or not to be distinguished; the extremities are cold, and the stomach is frequently affected with sickness. I have observed that when the movements of the chest, in the interval between the sighs, have been imperceptible or nearly so, the respiration has still been carried on by 
means of the diaphragm. It may also be remarked that the state of syncope is often relieved, for a time, by an attack of sickness and vomiting ; im. mediately after which the patient expresses himself as feeling better ; the countenance is somewhat improved, the breathing more natural, and the pulse stronger and more frequent.

In cases of fatal hæmorrhagy there are none of these ameliorations. The symptoms gradually and progressively assume a more and more frightful aspect. The countenance does not improve, but becomes pale and sunk; the consciousness sometimes remains until towards the last, when there is some delirium, but every thing denotes an impaired state of the energies of the brain. The breathing becomes stertorous, and at length gasping. The pulse is extremely feeble or even imperceptible. Animal heat fails, and the extremities become colder and colder in spite of every kind of external warmth; the voice may be strong, but there is constant restlessness and jactitation. Ultimately the strength fails, and the patient sinks, gasps, and expires.

From the state of syncope the system usually recovers itself spontaneously, if the cause by which the syncope was induced be discontinued. The principle by means of which this recovery is effected, may, without involving any hypothesis, be denominated re-action. This re-action of the 
Bystem may, under different circumstances, be ex: cessive or defective, or it may be destroyed altogether, each state leading to a corresponding series of phenomena. The description of these varied phenomena is reserved for the ensuing section. Previously however to leaving this part of our subject, it may be proper to notice that the brain is sometimes affected by loss of blood in a very different manner, and, instead of syncope, there are attacks of convulsion. Dr. Kellie observes that " fits resembling apoplexy and epilepsy, as well as fits of syncope, occasionally supervene to venesection at the arm "*; and Dr. Armstrong states that " bleeding to syncope in young children may sometimes lead even to fatal convulsions." +

II. Of the more remote or cumulative effects of repeated or protracted loss of blood, or Exhaustion.

The re-action or recovery from ordinary syncope is generally a simple return to a healthy state of the functions or nearly so, the pulse not passing beyond its natural frequency. In cases of profuse loss of blood, on the contrary, the recovery is not quite so uniform, and the pulse acquires and retains a morbid frequency for a certain length of time; this frequency of the pulse gradually subsides, however, and is unattended by

* Edinb. Med. Chir. Trans. Vol. I. p. 105.

+ On Puerperal Fever, 2d edit. p. 191. 
any other symptom of indisposition of any consequence. The phenomena are very different, if, instead of one full bleeding to syncope, or of a profuse hæmorrhagy, and even protracted syncope, the person be subjected to repeated blood-lettings or to a continued drain. In this case, within certain limits, the pulse, instead of being slow and feeble, acquires a morbid frequency and a throbbing beat, and there are, in some instances, all the symptoms of excessive re-action of the system, which it is my object now to describe.

The state of excessive re-action is formed gradually, and consists, at first, in forcible beating of the pulse, of the carotids, and of the heart, accompanied by a sense of throbbing in the head, of palpitation of the heart, and eventually perhaps of beating or throbbing in the scrobiculus cordis, and in the course of the aorta. This state of re-action is augmented occasionally by a turbulent dream, mental agitation, or bodily exertion. At other times it is modified by a temporary faintness or syncope. In the more exquisite cases of excessive re-action the symptoms are still more strongly marked. The beating of the temples is now accompanied by a throbbing pain of the head, and the energies and sensibilities of the brain are morbidly augmented. Sometimes there is intolerance of light, but still more frequently intolerance of noise and disturbances of any kind, requiring stillness to be strictly enjoined, the knockers to be tied, 
and straw to be strewed along the pavement. The sleep is agitated and disturbed by fearful dreams, and the patient is liable to awake in a state of great hurry of mind, sometimes almost approaching to delirium. In general this is slight, but occasionally severe and even continued. More frequently there are great noises in the head, as of singing, of crackers, of a storm, or of a cataract; in some instances flashes of light are seen. Sometimes there is a sense of great pressure or tightness in one part or round the head, as if the skull were pressed by an iron nail, or bound by an iron hoop.

The action of the heart and arteries is morbidly increased, and there occur great palpitation, and visible throbbing of the carotids, and sometimes even of the abdominal aorta, augmented to a still greater. degree, by every hurry of mind or exertion of the body, by sudden noises or hurried dreams and wakings. The patient is often greatly alarmed and impressed with the feeling of approaching dissolution. The state of palpitation and throbbing are apt to change, at different times, into a feeling of syncope. The effect of sleep is in some instances very extraordinary; sometimes palpitation, at other times a degree of syncope, or an overwhelming feeling of dissolution. The pulse varies from 100 to 120 or 130 , and is attended with a forcible jerk or bounding of the artery. 
'The respiration is apt to be frequent and hurried, and attended with alternate panting and sighing; the movement of expiration is sometimes obviously and singularly blended with a movement communicated by the beat of the heart; the patient requires the smelling-bottle, the fan, and fresh air. In this state of exhaustion, sudden dissolution has sometimes been the immediate consequence of muscular effort on the part of the patient.

The phenomena of excessive re-action are mostly observed in young persons of robust constitution, who have been subjected to repeated bloodletting. In infants, in feeble persons, and in rather advanced years, re-action after loss of blood is for the most part defective. In this case the patient long remains pale, thin, and feeble, and becomes faint on the slightest occasions; the pulse is frequent, but feeble and perhaps irregular, and we look in vain for the throbbing and palpitation observed in the young and robust. This state either gradually yields to returning strength, or subsides into the state of sinking. In the study of the effects of loss of blood it is particularly necessary to bear in mind this difference of the phenomena arising out of the previous state of the constitution, whether of vigour, or of feebleness.

The symptoms of exhaustion with excessive revol. XIII. 
action may gradually subside, and leave the patient feeble, but with returning health, or they may yield to the state of sinking. This term is adopted, not to express a state of negative weakness merely, which may continue long and terminate in eventual recovery, but to denote a state of positive and progressive failure of the vital powers, attended by its peculiar effects, and by a set of phenomena very different from those of exhaustion with re-action. In the latter the energies of the system were augmented; in the former the functions of the brain, the lungs, and the heart are singularly impaired.

The sensibilities of the brain subside, and the patient is no longer affected by noise as before. There is, on the contrary, a tendency to dozing, and gradually some of those effects on the muscular system, which denote a diminished sensibility of the brain, supervene, as snoring, stertor, blowing up of the cheeks in breathing, \&c.; instead of the hurry and alarm on awaking, as observed in the case of excessive re-action. The patient in the state of sinking requires a moment to recollect himself and recover his consciousness, is perhaps affected with slight delirium, forgets the circumstances of his situation, and, inattentive to objects around him, falls again into a state of dozing.

Not less remarkable is the effect of the state of exhaustion with sinking on the functions of the 
lungs. Indeed the very earlset indication of this state is, I believe, a crepitus in respiration, only to be heard at first on the most attentive listening. This crepitus gradually becomes more audible, and passes into slight rattling, heard in the situation of the bronchia and trachea. There is also a degree of labour or oppression in the breathing, inducing acuteness in the nostrils, which are dilated below, and drawn in above the lobes at each inspiration; in some cases there is besides, a peculiar catching laryngal cough, which is especially apt to come on during sleep, awaking the patient. The heart has, at the same time, lost its violent beat and palpitation, and the arteries their bounding or throb. bing. The stomach and bowels become disordered and flatulent, and the command over the sphincters is impaired. The last stage of sinking is denoted by a pale and sunk countenance, inquietude, jactitation, delirium, and coldness of the extremities*.

* I have already described, in a cursory manner, some of the effects of loss of blood, in two recent publications; the first in 1820, entitled "Cases of a Serious Morbid Affection arising from Irritation and Exhaustion"; the other in 1822, "An Essay on the Symptoms and History of Diseases", see chap. v. Since these periods I have seen several allusions to this important subject, and one especially by Mr. Cooke, in his useful and able abridgement of Morgagni. The observations of this author are highly valuable, and they have been confirmed by Dr. Kellie*, and, as far as the symptoms go, by myself.

Mr. Cooke observes, " after uterine hæmorrhage, and also

* Edinb. Med. Chir. Trans. Vol. 1. p. 105.

K 2 
The symptoms of exhaustion, first with re-action, but gradually passing into the state of sinking,

after copious depletion on account of pulmonary and other inflammations, I have frequently observed the symptoms of cerebral congestion, and which has generally appeared to arise from the excitement occasioned by some mental effort, though -occasionally it has arisen without an evident cause. Whilst the other parts of the body appear comparatively bloodless, the vessels of the head throb violently; there is severe pain; confusion of intellect sometimes to such a degree as to threaten delirium; the pulse at the wrist is usually small and vibrating, and the countenance distressed. When I first observed these symptoms I was led to abstract blood, from an apprehension of phrenitis, but I did harm, for, if the urgency of symptoms was diminished, the susceptibility to a recurrence was increased, and restoration to health protracted. The liability to this form of cerebral plethora has appeared to me to be proportionate to the preceding hemorrhage and the consequent debility. If, in this condition, an intrusive visitor be admitted to converse, though but for a short time, with the patient, or if the patient attempt to read, or in any other way to employ the mental faculties beyond what is perfectly easy, or if the mind be agitated, this state of the head will almost inevitably be induced. It may, however, be brought on by all those causes which tend to destroy the equilibrium of circulation, and none are more likely in this condition of the patient than noise in the room, deficiency of sleep, improper food, a constipated state of the bowels, or a morbid state of the secretions into them. This susceptibility to local congestion after excessive loss of blood, I presume depends upon the want of that due balance which, in a state of health, subsists between the nervous and vascular systems; but $I$ am jealous of hypotheses in medicine, and to pursue them in the present work would be unwarrantable." vol. r. p. 73.

"From the peculiarity of the conformation of the nose, epistaxis is sometimes a most uncontrollable form of hemor- 
are exemplified in a remarkable manner in the following case, the circumstances of which were more accurately noticed as they occurred in the person of a much respected friend and intelligent mem-

shage. I have recently seen two cases in which, if it were not absolutely the occasion of death, it certainly accelerated that event. The first was in a gentleman who laboured under hydrocephalus. He was a susceptible man, and would not endure a plug in the nostril. The hemorrhage frequently occurred; and when he had become excessively pallid from loss of blood, it was curious to observe to what an extent the irritative hemorrhagic action was propagated. $\Delta$ t first he only distinguished pulsation in and about the nose, but as his strength decreased, and his anxiety and susceptibility were heightened, the carotids could be seen throbbing vehemently, and a corresponding action was perceptible to the patient through their ramifications. The second case was in an old arthritic sufferer, who had been seized with cynanche parotidaca. The more acute inflammation had subsided, but the gland was much enlarged and indurated, and the jaw nearly rigid. In this state he was attacked with bleeding from the nose. It occurred sometimes when he was asleep, on which occasions he was threatened with suffocation from the formation of coagula in the fauces, which he removed with the utmost difficulty in consequence of being unable to open his mouth. The hemorrhage commenced in the nasal cavity nearest to the enlarged gland, but it afterwards took place from both nostrils. He was excessively afflicted with gout, and had indications of hepatic disorganization, but the immediate cause of death was the repcated effusion of blood. The hemorrhagic action was not so extensively manifest as in the preceding case, but when there was feebleness in the radial artery and the extremities were cold, the patient was conscious of a strongly irritative throbbing in the arteries ramifying through the nose and circumjacent parts." vol. 1. p. 110. 
ber of the profession, and principally under my own roof.

\section{$C A S E I$.}

Mr. C. C. aged forty, of an extremely muscular and robust make, was returning from Nottingham into the country on the $3 \mathrm{~d}$ of October 1821, when his horse reared, and fell backwards upon him, fracturing the third and fourth ribs of the left side. He was taken to an inn, and I saw him with a surgeon early on the following morning. He then suffered extreme pain of the side; there was a distinct crepitus but no emphysema; the face was somewhat bruised, swollen, and ecchymosed; the pulse was 100, and strong. Sixteen ounces of blood were taken from the arm, a dozen leeches were applied to the temples, and the same number over the fracture of the ribs; the motions of the chest were restrained by a tight bandage; calomel and purging medicines were freely given. At noon sixteen ounces of blood were again drawn from the arm, and a surcingle was applied round the chest.

During the whole of the 5th of October, the second day after the accident, $\mathrm{Mr}$. C. appeared to be going on well, but at night a violent attack of pain of the side induced him to bleed himself; this was done to syncope, and as a large wash-handbasin was used to receive the blood, its quantity 
was not known, but must have been very considerable. Seventeen leeches were then applied to the side and shoulder, with great relief. The surcingle, which had been removed, was re-applied, and the mercury and purgatives were continued. Early on the morning of the third day, there was another violent attack "of pain of the side with dyspnoa; a messenger was despatched for the medical attendants, but before their arrival Mr. C. had again bled himself, and taken away sixteen ounces of blood, unable to endure the pain. In another hour eight ounces more were drawn, the patient sitting upright; this was followed by syncope and great relief of pain.

On the fourth day, Mr. C. was removed a distance of about one mile to my house, and bore the journey on a litter extremely well, having previously lost a teacupfull of blood; he expressed himself as feeling better than at any time since the accident. In the evening an increase of pain took place, and about seven ounces of blood were taken with great relief. In all it would appear that $\mathrm{Mr}$. C. lost at least one hundred and twenty ounces of blood.

On the fifth day we were joined in consultation by an eminent physician and surgeon. There was much pain of the side, and it was at first proposed to take away more blood; but I had observed some of the symptoms which I knew to indicate 
re-action from exhaustion, and the venesection was omitted.

On the sixth day, the following circumstances were noticed. There was some degree of dyspnoea and of pain in the side, and the patient had removed the surcingle in the hope of obtaining relief; the mouth was affected with ptyalism. The pulse was 100, and had acquired a peculiar jerk; there was violent throbbing of the carotids, a pulsatory pain of the head, intolerance of noise, and, in a slight degree of light; at one time in the morning of this day great agitation had been induced by a knock at the door. On account of the intolerance of sound, the pavement was directed to be strewed with straw, and the ringing of the bells of the adjacent church discontinued. The bowels were freely moved: a draught, with tinctura opii and spiritus ammoniæ aromaticus, was given, with broth, arrow-root, sago, \&c.

Seventh day.-The patient was rather better towards evening yesterday. All the symptoms of strong re-action continued as before. The head has been much relieved by the application of a cold lotion.

On the succeeding day the pulse was 84 only, and had lost, in some degree, its peculiar jerk; the carotids beat less violently; the head was so much better as to render the lotion unnecessary; 
there was more tranquillity and some hilarity of mind. The aperients, the anodyne, and the nou rishment were continued.

I saw my patient about 3 o'clock a.m. on the ninth day, and I then heard the slightest degree of that crepitus in the breathing which I have already noticed as one of the first symptoms of sinking. The medical gentlemen met at nine, and the general symptoms were then so little changed that no degree of alarm was excited in their minds. I mentioned my fears, and the grounds on which they were formed. At this meeting cupping was proposed; but the changes in the patient were afterwards so rapid that brandy was recommended in the evening. The pulse was 110 , in the middle of the day, unattended by its previous force and jerk, and easily compressible ; the beating of the carotids had subsided; a slight degree of stupor was observed; on being left undisturbed the pa. tient fell asleep and snored; there was some labour in the respiration, and a troublesome, dry, laryngal cough; although the bandage was loose, there was no pain of the side of the chest; the countenance was anxious. The symptoms assumed a more alarming form during the day; at night there was considerable stupor, and, when the patient was roused, a degree of delirium; during this sleep there was much snoring and puffing up of the cheeks in expiration. On awaking he would feel greatly concerned that he should 
have appeared to blow in your face. There was much flatulence; the motions were extremely offensive, and passed at each attempt to void urine.

From midnight he could scarcely be roused, but if awoke he would speak collectedly, but in a hurried manner, and said he felt " such a dying feel ". - The pulse was about 120. At three o'clock a.m. I saw my patient; there was little change in the pulse or other symptoms, but in a minute or two the pulse became slow, feeble and irregular, he altered rapidly, and I found that he was moribund; in a few minutes more he expired.

On dissection the pleura was found morbidly red in the vicinity of the fracture, but not wounded; there was some effusion of lymph in its cavity. The right lung was found united to its contiguous pleura by old adhesions.

\section{$C A S E I I$.}

Mrs. Burrows, aged twenty-eight, of a stout constitution. After delivery there was uterine hæmorrhagy, which continued to recur for the twelve subsequent months. It was then discovered that Mrs. Burrows laboured under polypus uteri. A ligature was applied, purgative medicines given, and the patient presently recovered. The case is introduced in this place in order to present the de- 
tail of symptoms arising from a continued drain or loss of blood. There were, 1 . beating of the temples, a sense of "knocking" in the head, vertigo, dimness of sight, singing in the ears, terrific dreams, and starting from sleep. 2. Frequency of the pulse, pulsation of the carotids and aorta, fluttering and beating of the heart, faintishness, and a sense and fear of dissolution. The palpitation. of the heart on awaking was sometimes such as to move the bed-clothes, the bed, and, it is said, even the door. 3. The breathing was short and hurried, sometimes with panting, sometimes with sighing. 4. There were urgent calls for air, for opened windows, and the smelling-bottle; and the nostrils and temples were required to be bathed with sal-volatile or vinegar.

The countenance, prolabia, and tongue were pallid; the legs somewhat œdematous ; the bowels were irregular, the secretions morbid. Once there was obstinate constipation; frequently the bowels were merely confined, sometimes with sickness, but always with an increase of all the symptoms.

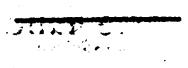

It would be difficult, perhaps, to offer any observations on the nature and cause of excessive re-action; but it is plain that the state of sinking involves a greatly impaired state of the functions of all the vital organs, and especially of the brain, 
from defective stimulus. The tendency to dozing, the snoring and stertor, the imperfect respiration, the impaired action of the sphincters, the defective action of the lungs, and the accumulation of the secretions of the bronchia, the feeble and hurried beat of the heart and pulse, the disordered state of the secretions of the stomach and bowels, and the evolution of flatus, all denote an impaired condition of the nervous energy. The state of sinking may, indeed, in certain points of view, be compared with the state of the functions in apoplexy, and with the effects observed on abstracting the influence of the brain and spinal 'marrow by dividing the eighth pair of nerves, or destroying the lower portion of the latter organ*.

III. On the effects of further loss of blood in cases of exhaustion.

The symptoms of exhaustion with re-action have, I am persuaded, frequently been mistaken for those of inflammation, or other disease of the head or of the heart. Under this impression recourse has frequently been had to the further detraction of blood by the lancet, and the effect of this practice is such as greatly to impose upon the inexperienced, for all the symptoms are perhaps fully relieved. It was some time before I could fully comprehend the nature of this fact.

* See Dr. Phillip's work on "The Vital Functions", passim. 
I had satisfied myself that, in certain cases, the symptoms were those of loss of blood, and yet it appeared no less certain that those very symptoms were relieved by the lancet. At length I discovered, by careful observation, that the symptoms which were relieved were those of reaction, and the mode of relief the substitution of syncope; that the relief endured as long as the state of faintishness continued, but returned as this state gave way to the rallying and re-action of the vital powers.

Another circumstance, equally interesting and curious, is, that within certain limits the remedy which relieves for a time, eventually only adds to the severity of the malady, which is apt to return after a certain period in a still more aggravated form. It is natural, indeed, to suppose that, unless where there is a tendency to failure of the vital powers, the re-action of the system and the painful circumstances attending it, would be greater after a third or fourth loss of blood, than after a first or second. Indeed there are seldom symptoms of re-action after one flow of blood, however great or profuse. The repetition or protraction of the cause is essential to produce this effect. It is observable, too, that in cases of exhaustion with re-action, syncope is very soon produced by the further loss of blood. This fact is of importance, because it may be regarded as a sign of the state of exhaustion when obscured by 
the re-action of the system, and as a warning voice against the further and inconsiderate use of the lancet.

If the loss of blood be repeated still further, not only syncope, but a state of sinking is induced ; the effects of re-action are, of course, in this case, permanently relieved; whilst a different series of phenomena, already fully described, is established. This transition of re-action into sinking may either be spontaneous, as in the case of Mr. C. C. detailed in the preceding section; or it may be the effect of a last bleeding, the state of syncope scarcely ceasing, with a total though gradual failure of the vital powers. These facts are illustrated by the following cases.

\section{CASE III.}

Mrs. Darker, aged twenty-one, of a rather feeble constitution, was confined of her first child. On the fifth day, the bowels having been constipated, she became much indisposed with flushing of the countenance, noise in the ears, as of a rushing wind or the explosion of crackers, flashes of light on lying down, beating of the carotids, $\& c$. , the pulse being 120 in a minute. Fourteen ounces of blood were taken from the arm, which induced deliquium with relief to the symptoms. About seven hours afterwards the noises in the head returned, and the pulse was 120 ; twelve 
ounces of blood were again drawn, and the patient again fainted. Eight ounces of blood were taken the next day. Early on the succeeding morning, the medical attendant was called; there had been little sleep, but much lowness for several hours; the patient then complained of violent beating in the head; the pulse was 120. A teacupfull of blood was taken, which induced faintishness and abated the beating of the head. By noon she was again flushed, and the beating had returned in an aggravated degree. From this period the patient was bled no more, but recovered under the influence of aperient and anodyne remedies.

\section{$C A S E I V$.}

Mrs. D., aged thirty-five, was confined in June, 1818. The expulsion of the placenta was followed by much hæmorrhagy, which induced great exhaustion. On the tenth day, she was seized with severe shivering, heat, throbbing pain of the head, and intolerance of light and sound. Ten ounces of blood were taken from the arm, about ten o'clock, a.m., which induced faintishness and gave relief. At seven o'clock in the evening, the pain of the head was as severe as ever, and twelve ounces of blood were taken from the arm. This was followed by dreadful faintness, and gasping breathing, so as even to lead to the apprehension of dissolution. On recovering, the pain and intolerance of light 
and sound returned as before. This patient became affected with all the symptoms of exhaustion with re-action, but gradually recovered without further venesection.

When the last bleeding has been considerable, it has, in some cases, been followed by the most dreadful gaspings and other convulsive motions, and death. It should be observed that between the most gradual sinking and the most sudden dissolution, as the effects of blood-letting, there is every intermediate shade, with the phenomena of which it is of the utmost importance to be acquainted. These varied phenomena may, I think, be collected from the observations which have been made in this and the preceding sections. They are further illustrated by the following cases which exemplify the fatal effects of loss of blood, as they supervene more or less gradually upon the use of the lancet.

\section{$C A S E \quad V$.}

MRs. — , aged thirty, had been affected with a slight attack of influenza. She.was seized with rigor, and soon afterwards the pains of labour came on. Delivery was effected in about fifteen hours, at nine o'clock, a.m., which was followed by much fever, the countenance being flushed, the pulse 
frequent, and the breathing difficult, with incessant cough. These symptoms increased towards evening and in the night, and about forty ounces of blood were drawn from the arm at two blood-lettings. The next morning twelve leeches were applied to the chest, with great relief. In the evening a blister was applied. The night was passed more comfortably : she dozed a little, and was cheerful, and continued relieved in the morn. ing. As a preventive against a relapse, however, three tea-cup-fulls of blood were taken. The patient became faint during the flow of the blood, sank from that time, and never again rallied. She became extremely feeble, and could scarcely articulate, and, from being cheerful the day before, was now impressed with the conviction of approaching dissolution, and expressed herself as unable to recover from the last bleeding. During this day, Saturday, and the two succeeding days, there was a state of extreme exhaustion, and still a sense of load at the chest, and pain of the side. On Tuesday the countenance was observed sometimes to flush to a deep scarlet, and then to become quite pallid, while a profuse perspiration frequently ran down the face. The pulse was extremely frequent, and the pain severe on coughing; there was no delirium, though she awoke hurried from sleeps which she described as "c just like death." During the four following days there was little obvious change; distressing faintings usually came on about two or three o'clock, p.m.

voL. XIII.

L 
On Sunday she became drowsy and evidently sinking, and she died in the evening of the succeeding day.

\section{$C A S E \quad V I$.}

Mrs. V., was of a pale sallow complexion, and weakly constitution. Six days before her confinement of her first child, she was awaked in the night by severe pain of the head confined to one spot. This pain continued several hours, when Mrs. V. applied to her medical man. She was completely relieved by losing sixteen ounces of blood, followed by purgative medicine, and she continued well. Mrs. V.'s labour occurred on the 1st of September, 1817, and was rather tedious, but natural, and she had no complaint until the second day, when she experienced a second attack of pain in the head, but less violent than the previous one. She was seen six hours after this attack; she then complained of pain and beating of the head, about the anterior part of the right parietal bone; the skin was hot, and the pulse frequent and strong. Sixteen ounces of blood were taken from the arm, leeches were ordered to be applied to the temples, and an enema and purgative medicine were prescribed. In three hours' time Mrs. V. was again visited, and it was deemed necessary to abstract more blood. Six or eight ounces were therefore taken; faintishness was induced, and the symptoms were abat- 
ed. On the succeeding morning, September the 4th, the symptoms still remained the same; the surface was hot; the bowels had been purged, and the evacuations were natural; the saline mixture was ordered. At noon the purgative medicine was repeated and a blister was applied. In the evening, the evacuation of the bowels was satisfactory; the pain of the head was not severe, but there was much beating, and a rushing noise, with restlessness and a teazing irritative cough. A draught, with thirty drops of the tinctura opii, was administered. The next morning, September the 5th, Mrs. V. expressed herself as feeling much better, having enjoyed comfortable sleep. The surface was still hot, and the head affected as before. In the evening there was a degree of tenderness in the region of the uterus. She dreaded the idea of being bled, from the faintishness she had before experienced from it, and said it would certainly kill her. On the morning of the 6th, the pain in the region of the uterus was relieved, but the head remained as before; the window was kept open for want of air. In the evening Mrs. V. complained of being faint and low. A mixture with camphor and sulphuric æther was prescribed. On the 7 th the irritative cough again occurred; the pulse was frequent, from 120 to 130 ; and the other symptoms remained unabated.

A physician was consulted. Sixteen ounces of blood were directed to be taken from the arm; a 
grain of calomel was given every three hours, and the effervescing medicine was ordered. On the morning of the $8 \mathrm{th}, \mathrm{Mrs}$. V. appeared to be relieved in every respect; the heat of surface and the pain of the head were diminished; the blood presented the buffy coat. It was thought proper to abstract more blood, as the last bleeding had apparently conferred benefit, and had been borne better than the preceding ones. Four tea-cupfulls of blood were taken. The most dreadful faintings followed, with gasping, open mouth, and a convulsive action of the diaphragm; and in an hour or two death closed the scene.

IV. Of the influence of various circumstances on the effects of loss of blood.

The first and principal circumstance which modifies the effects of loss of blood has reference to the strength of the patient. Cæteris paribus, the degree of re-action is proportionate to the degree of strength. In infancy, in declining years, and in those of feeble constitution, there is defective reaction after loss of blood, the phenomena of which have been already detailed. The state of syncope is then a state of danger, and a second or third blood-letting is borne with difficulty. In youth, and in the vigorous and robust, on the contrary, the re-action is strong, and especially marked after 
repeated venesections. In the strong, the state of sinking is even preceded by that of great re-acticn, unless indeed the strength be overwhelmed by the degree or early repetition of the evacuation. In the feeble it steals on insidiously and gradually; unmarked by re-action of the system.

The other circumstances which exert an influence on the effects of loss of blood, are certain states of disease. And I must, in this place, particularly remark, that the state of intestinal irritation leads to those effects of blood-letting which I have described as exhaustion; whilst inflammation seems to protect the system from the effects of loss of blood. In the former case throbbing is soon induced, unless indeed it be prevented by a state more nearly allied to syncope ; in the latter, bloodletting is followed by little of re-action until the state of inflammation be subdued, and the system freely exposed to the uncontrouled influence of loss of blood. In the former there is danger from full depletion ; in the latter, this measure is providentially not less safe than necessary.

In all cases we are only to expect the phenomena of re-action where a certain quantity of blood has been lost. One bleeding, although large, and even a continued drain, if not considerable, will not induce exhaustion, the powers of the system being sufficiently great to recruit and to restrain its actions. But exhaustion is sooner induced under 
circumstances of intestinal irritation, and less so under those of inflammation than in health; and re-action is the consequence, unless the strength of the patient be low, and then it is defective, or even gives way to a state of positive sinking. Each successive blood-letting is of course attended with increasing risk. There is considerable danger where the re-action is strong; still greater when it is feeble. A large blood-letting in such eases may be followed by sudden death. There is great danger when fainting has been several times induced, and where there is the least tendency to " want of air."

V. On the effects of loss of blood on the internal organs.

We are altogether in want of a series of observations on the effects of loss of blood on the internal organs. There is, I think, reason to suppose that a state of exhaustion from loss of blood may lead to effusion into the ventricles of the brain; and a

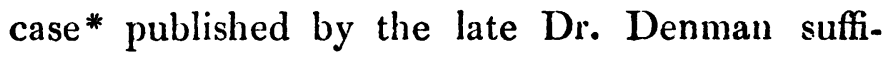
ciently proves that such a state of exhaustion is no protection against an attack of apoplexy. From these circumstances we may conclude that there is, even in cases of exhaustion from loss of blood, increased action or fulness of the vessels of the brain.

* Transactions of a Society for the Improvement of Medical and Surgical Knowledge, Vol. III. p. 315. 
The morbid state of the secretory function of the lungs in exhaustion with sinking has already been mentioned, and there is no question that, in protracted cases of this nature, the bronchia must become clogged, and the arterialization of the blood impeded. The state of flatulency, and the foetid evacuations of the intestines, sufficiently denote the morbid condition of this internal organ. There is also, in extreme cases of exhaustion, a general tendency to serous effusion, both into the internal cavities and into the cellular membrane. This effect of the loss of blood has been very long remarked by medical writers.

As I have carefully avoided, in this essay, the statement of any circumstance which I did not think amply substantiated by well-observed facts, I shall leave this part of my subject to be elucidated by future observation. I still have it in view to investigate the organic effects, and especially the remedies of loss of blood, by a series of experiments. 